June 5. Faint, ill-defined nucleus. - June 8. Fairly well defined nucleus. - June 17,24 . Round faint nebulous object; observed to condensed centre. - July 26. A planetary object of $7^{\mathrm{m}}$, well defined, surrounded by very faint coma. Aug. 4. Very faint object of undefined haze; occasional glimpses of a condensed centre. - Aug. 5, 7. Comet very faint. - April 26, May 2, 10, 2 r, 25, Aug. 5. Definition bad. The scale value and zero of the position circle were derived from observations of standard pairs of stars.

\title{
Beobachtung der totalen Mondfinsternis 1910 Mai 23 auf der Sternwarte Santiago de Chile.
}

\section{A. Visuelle Beobachtungen.}

Von den beiden Mondfinsternissen des Jahres 1910 wurde nur die vom 23 . Mai hier bei völlig günstigem Himmel beobachtet. Die zweite Finsternis vom I6. November, bei welcher der Mond erst im Augenblicke des Beginns der Totalität sich über die Cordillere erhob, hatte unter Wolken zu leiden, welche nur einzelne Momente und von den durch die Sternwarte Pulkowa angezeigten Sternbedeckungen nur eine unsicher $z$ u beobachten erlaubten, so daß ich es vorziehe, die erhaltenen Resultate gänzlich zu unterdrücken.

Für die Finsternis am 23. Mai waren die Instrumente wie folgt verteilt. Ristenpart: Äquatorial Young, Öffnung $162 \mathrm{~mm}$, Vergrößerung 30; Prager: Refraktor von Repsold,
Öffnung $241 \mathrm{~mm}$, Vergrößerung 75. Herr Dr. Zurhellen bediente sich, da das astrophotographische Fernrohr bereits für den Transport nach Lo Espejo auseinandergenommen war, einer einfachen Holzkamera, die er auf die Deklinationsachse des Äquatorials Fraunhofer aufgesetzt hatte, und mit der er schon gut gelungene Photographien des Kometen Halley erhalten hatte. Über die Aufnahmen und deren Ausmessung berichtet er selbst im zweiten Teile dieses Aufsatzes. Mit den beiden andern Fernrohren wurden folgende in mittlerer Zeit Santiago angesetzte Momente beobachtet, wobei den von Bruns A. N. 3065 angezeigten Kratern der Vorzug gegeben wurde:

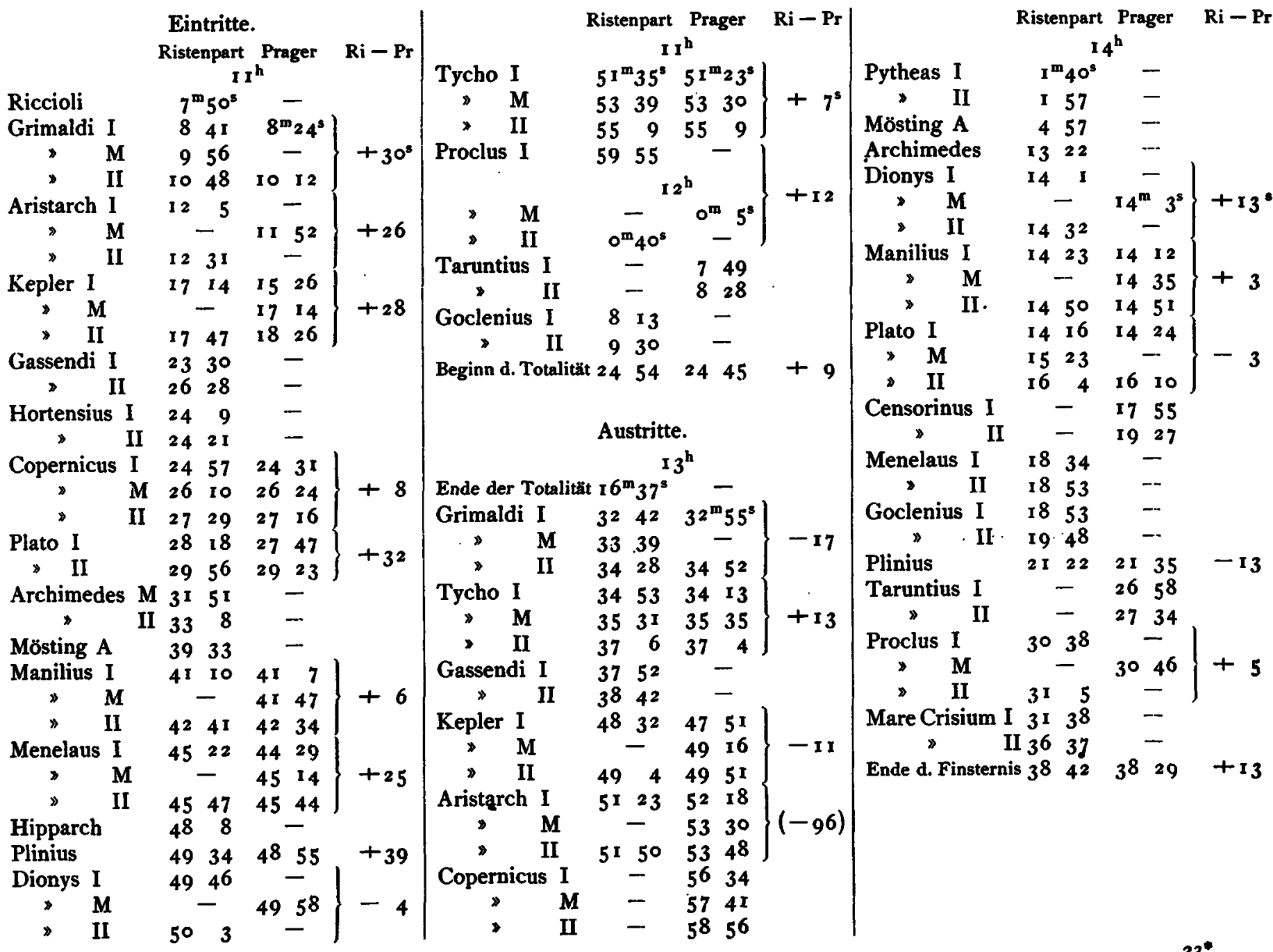


Bildet man für die gemeinsam beobachteten Punkte die Differenz $\mathrm{Ri}-\mathrm{Pr}$, und zwar für diejenigen Krater, die an mehreren Stellen beobachtet wurden, auf die Mitte reduziert, so ergibt sich, wenn allen Differenzen gleiches Gewicht gegeben wird, für die Eintritte:

$$
\mathrm{Ri}-\mathrm{Pr}=+18^{\mathrm{s}} \pm 4^{\mathrm{s}}
$$

für die Austritte (mit Ausschluß von Aristarch):

$$
\mathrm{Ri}-\mathrm{Pr}=\mathrm{o}^{\mathrm{s}} \pm 4^{\mathrm{s}} \text {. }
$$

Für die Eintritte scheint demnach eine systematische Differenz verbürgt werden zu können, die darin ihre Ursache haben mag, daß Pr die Eintritte durch ein vorgeschaltetes rotes Glas beobachtete, mithin die Verfinsterungen zu früh notierte. Die Austritte mußte er ohne dieses Glas beobachten, da sonst die Details der Mondoberfläche verschwanden und das Herannahen der Krater an die Schattengrenze nicht rechtzeitig genug bemerkt werden konnte.

$$
\text { Santiago de Chile, rgro Dez. } 17 .
$$

\section{B. Photographische Aufnahmen.}

Die während der Finsternis ausgeführte Serie von Aufnahmen war ursprünglich nur zu instruktiven Zwecken, nicht mit der Absicht, exakte Beobachtungen zu gewinnen, unternommen worden. Das Instrument, das sonst zu Aufnahmen des Halleyschen Kometen diente, war eine einfache Holzkamera mit einem alten Portraitobjektiv geringer Qualität, dessen Brennweite rund $45 \mathrm{~cm}$ beträgt; sein Objektiv (gewöhnlich $10.5 \mathrm{~cm}$ Durchmesser) war auf $3 \mathrm{~cm}$ abgeblendet, so daß ein kurzes Lüften des Objektivdeckels, also eine Exposition von etwa 0.5 , auf einer Platte von mittlerer Empfindlichkeit (Lumière blau) die richtige Stärke des Bildes gab. Auf die Visierscheibe der Kamera hatte ich ein quadratisches Netz von 49 je I cm voneinander entfernten Schnittpunkten aufgetragen und stellte die Mondscheibe sukzessive hierauf ein. Dieses erforderte ein ständiges Wechseln von Visierscheibe und Kassette, das ich gern vermieden hätte; doch bot die primitive Montierung der Kamera keine andre Möglichkeit der Einstellung. Die Regelmäßigkeit der Anordnung gelang zwar nicht streng, aber leidlich; ebenso die Gleichmäßigkeit der Expositionen. - Von den 49 Bildern sind 24 vor, 24 nach der Totalität (von letzteren eins mißlungen), und eins, das mittelste, während der Totalität aufgenommen. Auf Gleichheit der Zeitintervalle konnte nicht die erwünschte Rücksicht genommen werden, da die Schwierigkeit der Einstellung in der Nähe der Totalität erheblich wuchs.

Die bei fast allen Bildern unerwartet hohe Schärfe des beleuchteten Mondrandes veranlaßte mich zu dem Versuch einer Ausmessung mit dem Doppelmikrometer des Repsoldschen Meßapparats, wozu sich die Bilder auch ihrem Durchmesser (etwa $3.8 \mathrm{~mm}$ ) nach vortrefflich eigneten. In der Tat bestätigte es sich, daß der an den Rändern begangene Meßfehler gegenüber der Unsicherheit der Schattenkontur völlig verschwindèt; der mittlere Fehler der Messung eines Durchmessers, soweit eine solche möglich war, verhält sich zu dem der übrigen Distanzen, bei denen mindestens eine Schattenkontur eingestellt war, etwa wie $1: 7$ oder I : 8 . Damit scheint mir die Brauchbarkeit selbst so kleiner und mit so primitiven Mitteln gewonnener Photogramme zur Ableitung exakter Beobachtungsdaten erwiesen zu sein. Bisher der Totalität notiert:

Außerdem wurden folgende Sternbedeckungen während

$$
\begin{aligned}
& \text { Eintritte M. Z. Santiago. } \\
& \text { BD Ristenpart Prager } \\
& -20^{\circ} 439 \mathrm{I} \quad \text { I } 2^{\mathrm{h}} 28^{\mathrm{m}} \quad 4^{\mathrm{s}} 6 \\
& \begin{array}{llll}
-20^{\circ} 4392 & \text { I } 2 & 29 & 47 \\
- & \text { I }
\end{array} \\
& -20^{\circ} 4397 \text { I } 3 \text { I } 14.0 \\
& -20^{\circ} 4400 \quad \text { I } 3 \text { 10 } 22.5 \\
& \begin{array}{lll}
-20^{\circ} 4401 & 132049.0
\end{array}
\end{aligned}
$$

Die Positionen der Sterne werden in dem Katalog der Anhaltsterne der astrographischen Platten enthalten sein, der hier am Meridiankreis in Beobachtung ist.

Der Mond war während der Finsternis in kupferrotem Lichte sichtbar, nur der südliche Rand, der nahe an der Schattengrenze lag, war mehr grau.

\section{F. W. Ristenpart.}

ist meines Wissens eine regelrechte Ausmessung von Finsternisaufnahmen noch nicht publiziert worden, so daß die hier vorgelegten Resultate nur mit optischen Beobachtungen verglichen werden können.

Ein Nachteil der photographischen Methode liegt zweifellos hier, wie auch sonst, in dem relativ erheblichen Arbeitsaufwand, den eine exakte Ausmessung und Reduktion erfordert; ein zweiter in dem Umstand, daß eine Verwandlung von Meßeinheiten in Bogenmaß nötig wird und diese in den Fällen, in denen die Messung des Monddurchmessers unmöglich ist, eine gewisse Unsicherheit in die Resultate hineinträgt. Trotzdem scheint mir die photographische Beobachtung des Schattens neben der optischen von Wichtigkeit zu sein, da der Schatten bekanntlich photographisch sehr lichtschwach ist (vgl. z. B. die Untersuchungen von Montangerand, C. R. 128 etc.) und die Vergleichung der Resultate der beiden unabhängigen Beobachtungsmethoden zu wertvollen Schlüssen führen könnte. Die oben erwähnte mittelste Aufnahme $7^{\text {m }}$ nach Eintritt der Totalität, die auf $30^{\circ}$, also etwa das 60 fache der übrigen Expositionen ausgedehnt wurde, ergab gegen meine Erwartung nur eine dünne schwache Sichel an der Stelle, die zuletzt noch direktes Licht gehabt hatte.

Von jedem Bild sind folgende Daten in zwei um $180^{\circ}$ verschiedenen Lagen gemessen: a) mit der $x$-Schraube senkrecht zur Hörnersehne die relativen Abstände von Hörnersehne, Schattengrenze und beleuchtetem Rand; b) mit der $y$-Schraube die Länge der Hörnersehne und, sofern das möglich war, der hierzu parallele Durchmesser. Die Ganghöhe der beiden Schrauben war als sehr nahe identisch, ihre Senkrechtstellung als einwandfrei bekannt. Von dem Schattensaum wurde immer das äußerste Ende der Schwärzung der Platte, also die äußerste Grenze des photographisch wirksamen Lichtes oder der Anfang der völligen (photographischen) Verfinsterung eingestellt. Positionswinkel zu messen wäre zwecklos und irreführend gewesen, da die Orientierung der Bilder zahlreichen unkontrollierbaren Fehlerquellen ausgesetzt war. - Die Kolumnen 3-6 der Tabelle enthalten die Messungsresultate, d. h. die unter a) und b) angegebenen Längen in einer Einheit gleich einer halben Schrauben- 
revolution $=$ etwa $1 / 6 \mathrm{~mm}=$ etwa $77^{\prime \prime} ;$ unten ihre mittleren Fehler, berechnet aus der Quadratsumme der Abweichungen der beiden Lagen, von denen eine Konstante abgesondert war. Zur Verwandlung der Strecken in Bogenmaß diente für die Bilder I-I 3, 36-49 natürlich der gemessene Durchmesser, dessen Bogenwert bekannt war:

Anfang der Finsternis I 774.6 Ende der Totalität I 775.3 》Totalität I 775.0 \# Finsternis I 775.8 .

Eine Prüfung der gemessenen Durchmesser zeigte nun, daß für die übrigen Bilder, I 4-35, keineswegs ein konstanter Wert angenommen werden konnte, daß vielmehr eine Veränderlichkeit des Einheitswertes über die Platte hin deutlich ausgesprochen war. Ich habe infolgedessen die gemessenen Werte unter Annahme einer quadratischen Funktion mit 6 Konstanten nach einem vereinfachten Näherungsverfahren ausgeglichen, indem ich als Koordinaten der Bilder die runden Werte annahm, die sie (in Zentimetern) haben sollten: Serie $1-7$ erhielt $y=+3$, und zwar Bild I $x=-3$, bis Bild $7 x=+3$; Serie 8-1 $4 y=+2, x$ analog; u.s.w. bis Serie $43-49 y=-3$, Bild $43 x=-3$, Bild $49 x=+3$. So erhielt ich :

$$
\begin{aligned}
\text { Durchmesser }= & -0.0134 x^{2}+0.0229 y^{2}-0.0142 x y \\
& -0.0010 x+0.045^{2} y+22.923 .
\end{aligned}
$$

Die so berechneten Durchmesser, von den gemessenen subtrahiert, geben als mittleren Fehler $m= \pm \sqrt{[v v]:(27-6)}$ $= \pm 0.073$. Dieser ist als m. F. der Durchmesser anzusehen, die für die Bilder 14-35 nach der Formel berechnet wurden und in der Tabelle eingeklammert sind. Für die übrigen, $I^{-1} 3$ und $36-49$ gilt hingegen der Wert \pm 0.022 . Die
Differenz der beiden besagt, daß die quadratische Funktion die Messungen nicht vollständig darstellt, daß vielmehr noch unregelmäßige Einflüsse, Schlotterung der Kassette, Fokusänderungen des Objektivs oder dergl. anzunehmen sind. (Der Wert $\pm 0.07 \overline{3}$ käme einer Verschiebung der Platte aus der Brennebene um $\pm \mathbf{1 . 4} \mathrm{mm}$ gleich, welchen Betrag ich nicht für wahrscheinlich halte). Da somit der mittlere Fehler der eingeklammerten Zahlen schon nahezu die Hälfte desjenigen der an den betreff. Bildern ausgeführten Distanzmessungen beträgt, und überdies diese Bilder weniger gut im Fokus sind als die äußeren Serien, so verdienen die Resultate von 1 4-35 nur etwa $2 / 3$ des Gewichts der übrigen.

Aus diesen gemessenen oder berechneten Durchmessern wurde der Einheitswert bestimmt (7. Kolumne; I $4-35$ wieder eingeklammert); hiermit wurden die Messungen in Bogensekunden verwandelt (Kolumne 8-10). Durch die Verwandlung erhöht sich der mittlere Fehler für I-I 3, 36-49 kaum um O." I, für $14^{-35}$ dagegen $u m$ etwa $I^{\prime \prime}$, so daß man hier rund $\pm 4^{\prime \prime}$ anzunehmen hat. - Zur Vergleichung mit optischen Beobachtungen wird man annehmen dürfen, daß die durchschnittliche Geschwindigkeit der Vorwärtsbewegung des Schattens nicht wesentlich von derjenigen abweicht, die man erhält, wenn man den Monddurchmesser durch die Dauer der Partialität dividiert. Im vorliegenden Fall ist also $\pm \mathrm{r}^{\prime \prime}$ Schattenwanderung äquivalent mit $\frac{82^{\mathrm{m}}}{\mathrm{I} 775}$ oder 2.75 . Die ersten beiden unter der Tabelle aufgeführten mittl. Fehler sind also äquivalent mit $3^{6}$ resp. $34^{5}$. Ein Äquivalent für

\begin{tabular}{|c|c|c|c|c|c|c|c|c|c|}
\hline \multirow[b]{2}{*}{ Nr. } & \multirow[b]{2}{*}{ M. Z. Santiago } & \multirow{2}{*}{$\begin{array}{l}\text { Hörn.-S. } \\
\text { - Rand }\end{array}$} & \multicolumn{3}{|c|}{ Gemessen } & \multirow{2}{*}{$\begin{array}{c}1 \text { Einheit } \\
=\end{array}$} & \multicolumn{3}{|c|}{ In Bogensekunden verwandel } \\
\hline & & & $\begin{array}{l}\text { Schatt-Gr. } \\
\text { - Rand }\end{array}$ & Hörn.-S. & Durchm. & & $\begin{array}{l}\text { Hörn.-S. } \\
\text { - Rand }\end{array}$ & $\begin{array}{l}\text { Sch.-Gr. } \\
\text { - Rand } \\
\end{array}$ & Hörn.-S. \\
\hline I & I I ${ }^{\mathrm{h}} 9^{\mathrm{m}} 32^{\mathrm{s}}$ & 20.574 & I 9.420 & I 2.912 & 23.080 & 76.89 & $\mathrm{I} 582^{\prime \prime}$ & $1493^{\prime \prime}$ & $993^{\prime \prime}$ \\
\hline 2 & I I $\quad$ I $3 \quad 9$ & 20.042 & I 8.702 & I 4.243 & 23.232 & 76.39 & I 53 I & 1429 & I $\circ 88$ \\
\hline 3 & I I $\quad$ I $6 \quad 24$ & 19.578 & 17.806 & I 5.268 & $23 \cdot 312$ & 76.13 & 1490 & I $35^{6}$ & 1162 \\
\hline 4 & $\begin{array}{llll}\text { I } 1 & \text { I } 8 & \text { I } 5\end{array}$ & $\mathrm{I} 8.8 \mathrm{I} \mathrm{I}$ & 17.059 & I $6.95^{8}$ & $23.29 \mathrm{I}$ & 76.19 & 1433 & 1300 & 1292 \\
\hline 5 & I I $20 \quad 29$ & 17.825 & I 6.1 I 8 & I 7.793 & $23 \cdot 178$ & 76.57 & I 365 & I 234 & 1362 \\
\hline 6 & I $1223^{8}$ & 17.872 & 15.916 & I 8.165 & 23.166 & 76.61 & I 369 & I 2 I9 & I 392 \\
\hline 7 & $\begin{array}{lll}\text { I I } & 24 & 49\end{array}$ & $17 \cdot 137$ & 14.844 & 19.178 & 22.990 & 77.19 & I 323 & $\times 146$ & 1480 \\
\hline 8 & $\begin{array}{lll}11 & 27 & 37\end{array}$ & I 6.789 & I 4.639 & 19.345 & 23.189 & 76.53 & I 285 & I I 20 & I 481 \\
\hline 9 & I I $29 \quad 3$ I & I 5.838 & $\mathrm{I} 3.49 \mathrm{I}$ & I 9.834 & 2.3 .145 & 76.68 & I 2 I 4 & 1035 & $152 \mathrm{I}$ \\
\hline IO & $\begin{array}{lll}\text { I I } & 3 \mathrm{I} & 4 \mathrm{I}\end{array}$ & I 5.404 & 13.047 & 20.347 & 23.192 & 76.53 & I 179 & 998 & I 557 \\
\hline I I & I I $33 \quad 38$ & I 4.935 & I $2.4^{6} 3$ & 20.449 & 23.122 & 76.76 & I I 46 & 957 & $\times 570$ \\
\hline I 2 & I I $\quad 36 \quad 28$ & I 3.898 & I 1.492 & 21.380 & 22.986 & $77.2 \mathrm{I}$ & 1073 & 887 & $165 x$ \\
\hline I 3 & I I $39 \quad$ I 5 & I 3.625 & I 1.240 & 21.809 & 23.025 & 77.08 & $105^{\circ}$ & 866 & 1681 \\
\hline I 4 & I I 4 I 52 & $12.3 \times 9$ & $9.9 \times 1$ & $22.23^{8}$ & $(22.896)$ & $\left(77 \cdot 5^{2}\right)$ & 955 & 768 & 1724 \\
\hline 15 & I I $46 \quad 23$ & I 1.405 & 9.026 & 22.173 & $(22.916)$ & $(77.45)$ & $88_{3}$ & 699 & 1717 \\
\hline 16 & I I $48 \quad 46$ & 10.436 & $8.2 \mathrm{I} \mathrm{I}$ & 21.931 & $(22.967)$ & $(77.28)$ & 807 & 635 & 1695 \\
\hline I 7 & I I $50 \quad 53$ & 9.865 & 7.667 & $21.75^{2}$ & $(22.993)$ & $(77.19)$ & 762 & 592 & 1679 \\
\hline I 8 & I I $53 \quad 5^{2}$ & $8.97 \mathrm{I}$ & $6.7 \mathrm{I} 4$ & $21.4 \circ 3$ & $(22.99 \mathrm{I})$ & $(77.20)$ & 693 & 5 I 8 & 1652 \\
\hline 19 & I I $57 \quad 2$ & 7.938 & 6. I I I & 20.707 & $(22.963)$ & $(77.29)$ & 614 & 472 & 1601 \\
\hline 20 & I I 59 I 9 & $7 \cdot 395$ & $5 \cdot 314$ & 20.276 & $(22.907)$ & $(77.48)$ & 573 & 4 I 2 & $157 \mathrm{I}$ \\
\hline $2 \mathrm{I}$ & $\left.\begin{array}{lll}12 & 2 & 4\end{array}\right)$ & 6.433 & $4.46 \mathrm{I}$ & 10.344 & $(22.824)$ & $(77.76)$ & 500 & 347 & I 504 \\
\hline 22 & 632 & 5.178 & 3.625 & 1 7.663 & $(22.805)$ & $(77.83)$ & 403 & 282 & I 375 \\
\hline 23 & $\begin{array}{lll}12 & 9 & 37\end{array}$ & 4.487 & 3.068 & I 6.468 & $\left(\begin{array}{lll}2 & 2.87 & 1\end{array}\right)$ & $(77.6 \mathrm{I})$ & 348 & $23^{8}$ & I 278 \\
\hline 24 & I 2 I 456 & $3 \cdot 555$ & 2.489 & I 3.146 & $(22.9$ I I $)$ & $\mid(77.47)$ & 275 & I 93 & IOI 9 \\
\hline
\end{tabular}
den Fehler der Hörnersehne kann nicht angegeben werden. 


\begin{tabular}{|c|c|c|c|c|c|c|c|c|c|c|c|}
\hline \multirow{2}{*}{ Nr. } & \multirow{2}{*}{\multicolumn{3}{|c|}{ M. Z. Santiago }} & \multirow{2}{*}{$\begin{array}{l}\text { Hörn.-S. } \\
\text { - Rand }\end{array}$} & \multicolumn{3}{|c|}{ Gemessen } & \multirow{2}{*}{$\begin{array}{c}\text { I Einheit } \\
=\end{array}$} & \multicolumn{3}{|c|}{ In Bogensekunden verwandelt } \\
\hline & & & & & $\begin{array}{c}\text { Schatt.-Gr. } \\
- \text { Rand } \\
\end{array}$ & -Hörn.-S. & Durchm. & & $\begin{array}{l}\text { Hörn.-S. } \\
\text { - Rand } \\
\end{array}$ & $\begin{array}{l}\text { Sch.-Gr. } \\
- \text { Rand } \\
\end{array}$ & Hörn.-S. \\
\hline $\left.25^{2}\right)$ & & - & & - & - & - & $(22.923)$ & $\left(77^{\prime \prime} \cdot 44\right)$ & - & - & - \\
\hline 26 & $13^{\mathrm{h}}$ & $28^{\mathrm{m}} 3$ & $3 r^{5}$ & 3.140 & $\mathrm{I} .8 \mathrm{I} \mathrm{I}$ & I 3.880 & $(22.909)$ & $(77.49)$ & $243^{\prime \prime}$ & I $40^{\prime \prime}$ & $1076^{\prime \prime}$ \\
\hline $\left.27^{3}\right)$ & & - & & - & - & - & $(22.867)$ & $(77.64)$ & - & - & $-\cdot$ \\
\hline 28 & I 3 & 393 & 37 & 5.982 & 3.893 & I 9.064 & $(22.799)$ & $(77.87)$ & 466 & $3 \circ 3$ & 1485 \\
\hline 29 & & 444 & 45 & 7.865 & 5.653 & 20.478 & $(22.740)$ & $(78.07)$ & 6 I 4 & $44 \mathrm{I}$ & I 599 \\
\hline 30 & I 3 & 473 & & 8.609 & 6.353 & 21.035 & $(22.82 \mathrm{I})$ & $(77.80)$ & 670 & 494 & 1636 \\
\hline $3^{I}$ & I 3 & $5^{\circ}$ & & 9.165 & $7.02 \mathrm{I}$ & 21.249 & $(22.875)$ & $(77.6 \mathrm{I})$ & $7 \mathrm{II}$ & 545 & 1649 \\
\hline 32 & I 3 & $5^{2}$ & 9 & 9.570 & 7.533 & $2 \mathrm{I} .54 \mathrm{I}$ & $(22.901)$ & $(77 \cdot 53)$ & 742 & 584 & 1670 \\
\hline 33 & I 3 & 543 & & 10.686 & 8.426 & 22.016 & $(22.901)$ & $(77 \cdot 53)$ & 829 & 653 & 1707 \\
\hline 34 & I 3 & 574 & & I I. 664 & 9.683 & $22.25^{2}$ & $(22.873)$ & $(77.62)$ & $9 \circ 5$ & $75^{2}$ & 1727 \\
\hline 35 & I 4 & I & & I 2.396 & 10.190 & 22.522 & $(22.820)$ & $(77.80)$ & 964 & 793 & $175^{2}$ \\
\hline $3^{6}$ & I 4 & 42 & $2 \mathrm{I}$ & I 3.574 & I I . 4 I 4 & 22.086 & 22.670 & 78.32 & 1063 & 894 & $173^{\circ}$ \\
\hline 37 & 14 & 7 & 9 & I 4.038 & I I .588 & 21.863 & $22.7^{6} 3$ & 78.00 & 1095 & 904 & $17 \circ 5$ \\
\hline $3^{8}$ & I 4 & 8 & 53 & 14.622 & I 2.229 & 2 I.344 & 22.793 & 77.90 & I 1.39 & 95.3 & 1663 \\
\hline 39 & $\mathrm{r} 4$ & IO & 27 & 15.218 & I 3.026 & 20.926 & 22.922 & $77 \cdot 46$ & I 179 & 1009 & 1621 \\
\hline 40 & $\mathrm{r}_{4}$ & I 2 & 10 & I 5.883 & I 3.629 & $20.54 \mathrm{I}$ & 22.924 & $7 \cdot 7 \cdot 46$ & 1230 & $1 \circ 56$ & I $59 \mathrm{I}$ \\
\hline 4 I & I 4 & 14 & I & 16. 106 & I 3.894 & 20.244 & 22.868 & 77.65 & $125^{\mathrm{r}}$ & 1079 & I 572 \\
\hline 42 & I 4 & I 6 & 6 & I 6.629 & I 4.569 & 20. I I I & 22.926 & 77.45 & I 288 & I I 28 & I $55^{8}$ \\
\hline 43 & I 4 & I 8 & 28 & I 7.493 & Y 5.355 & I 9. I I 4 & 22.8 IO & 77.85 & 1362 & I 195 & I 488 \\
\hline 44 & I 4 & $2 \mathrm{I}$ & 34 & 18.319 & I 6.235 & I 7.805 & $22.95^{8}$ & $77 \cdot 35$ & 1417 & I 256 & I 377 \\
\hline 45 & I 4 & 23 & & 18.865 & I 6.808 & I 7.394 & 22.975 & 77.29 & I $45^{8}$ & I 299 & I 344 \\
\hline 46 & I 4 & 25 & 12 & I 8.988 & I 7.188 & I 6.694 & 22.988 & 77.25 & 1467 & I 328 & I 290 \\
\hline 47 & 14 & 27 & 40 & I 9.569 & 17.833 & I 5.318 & $23.05 \mathrm{I}$ & 77.04 & I 508 & I 374 & I I 80 \\
\hline $4^{8}$ & I 4 & $3 \mathrm{I}$ & I 7 & 20.679 & I 9.794 & I $2.4 \mathrm{I} 2$ & $23.05^{\circ}$ & 77.04 & I 593 & 1525 & $95^{6}$ \\
\hline \multirow[t]{2}{*}{49} & I 4 & $3^{6}$ & I & 21.246 & 20.718 & $10.93^{\circ}$ & 22.973 & 77.30 & 1642 & 1602 & 845 \\
\hline & & & & \pm 0.170 & \pm 0.158 & \pm 0.176 & $\begin{array}{l} \pm 0.022 \\
( \pm 0.073)\end{array}$ & & $\pm 13 " 2$ & \pm 12.2 & \pm 13.7 \\
\hline
\end{tabular}

1) Unsicher bis $z u+10^{5}$

2) Totalität.

i) Mehrfaches Bild, ganz unsicher.

Optisch (mit Opernglas) beobachtet: Eintritt der völligen Totalität (Ende des Saumes) $2^{\mathrm{h}} 27^{\mathrm{m}} \cdot 6$; F.nde der Finsternis (Saum noch auf der Mondscheibe) $14^{\mathrm{m}} 3^{8 \mathrm{~m}} \cdot 2$.

Santiago, I 9 I 0 August.

Dr. W. Zurhellen.

\section{Observation de l'éclipse de Lune 1910 Nov. 16 à l'Observatoire de Lisbonne (Tapada).}

L'éclipse totale de Lune du i 6 Novembre i 9 Io devant être visible dans de bonnes conditions à Lisbonne, M. le Directeur Campos Rodrigues me chargea de l'observer à notre réfracteur de I 4 pouces (le seul qui pût discerner des étoiles aussi faibles) par la méthode déjà tentee ici plusieurs fois, mais non encore réalisée sans contrariétés provenant du mauvais temps, et qui se trouve décrite dans A. N. 138. 1 7 et 165.193. En outre on comptait aussi pouvoir observer l'arrivée de l'ombre aux différents cratères, ou autres points remarquables du disque lunaire.

Après que nos préparatifs étaient déjà assez avancés, - tels que le calcul de la position apparente du centre de la Lune de 2 en 2 minutes de temps sidéral, et la construction de la carte à l'échelle de 4 millimètres pour une minute d'arc - est arrivée ici, le 30 Octobre, la circulaire de $M$. Wittram, nous invitant à cette observation, et indiquant trois étoiles de la $\mathrm{BD}$ qui seraient occultées pour nous pendant la totalité. Ces étoiles étaient donc portées d'avance sur la carte.
L'observation a été réalisée, cependant, par un temps peu favorable: au lever de la Lune et jusqu'aux environs de l'entrée dans l'ombre le ciel était splendide et promettait une belle nuit; puis il commença à se couvrir d'alto-cumulus, qui bientôt, chassés par un vent de WNW s'épaissirent au point d'empêcher toute observation, et même de voir la Lune. Peu avant la fin de la totalité il commença même à pleuvoir, aucune nouvelle éclaircie ne s'étant plus produite. Dans ces conditions il est clair que les occultations ne furent observées que par hasard, ainsi que l'arrivée de l'ombre aux cratères, l'identification de ceux-ci étant d'ailleurs rendue difficile par leur peu de visibilité à travers les nuages et leur complète disparition après être éclipsés.

Dans les rares moments où des éclaircies entre les nuages permettaient de voir la Lune à découvert, on a pu noter que la couleur du disque était d'un rouge brique fort clair, et que les bords se sont toujours conservés plus clairs encore. En outre, on a pu noter dans la lunette un fait curieux: l'ombre portée des pics de la chaìne des Apennins 